\title{
Nanoparticle Enhanced Plasmonic Biosensor for Digital Biomarker Detection in a Microarray
}

Alexander Belushkin, Filiz Yesilköy and Hatice Altug

Institute of BioEngineering, École Polytechnique Fédérale de Lausanne, CH-1015 Lausanne, Switzerland

Corresponding Author:

*E-mail: hatice.altug@epfl.ch

\section{ABSTRACT}

Nanoplasmonic devices have become a paradigm for biomolecular detection enabled by enhanced light-matter interactions in the fields from biological and pharmaceutical research to medical diagnostics and global health. In this work, we present a bright-field imaging plasmonic biosensor that allows visualization of single sub-wavelength gold nanoparticles (NPs) on large-area gold nanohole arrays (Au-NHAs). The sensor generates image heatmaps that reveal the locations of single NPs as high-contrast spikes, enabling the detection of individual nanoparticle-labeled molecules. We implemented the proposed method in a sandwich immunoassay for the detection of biotinylated bovine serum albumin (bBSA) and human C-reactive protein (CRP), a clinical biomarker of acute inflammatory diseases. Our method can detect $10 \mathrm{pg} / \mathrm{ml}$ of bBSA and $27 \mathrm{pg} / \mathrm{ml} \mathrm{CRP} \mathrm{in} 2$ hours, which is at least four orders of magnitude lower than the clinically relevant concentrations. Our sensitive and rapid detection approach paired with the robust large-area plasmonic sensor chips, which are fabricated using scalable and low-cost manufacturing, provides a powerful platform for multiplexed biomarker detection in various settings.

Keywords: biosensors, gold nanoparticles, plasmonic nanohole arrays, plasmonic imaging, digital molecule detection, sepsis biomarker detection.

Nanophotonics is offering multiple opportunities to realize high performance biosensors for the fields ranging from biological and pharmaceutical research to medical diagnostics and global healthcare. ${ }^{1-7}$ For instance, the management of acute diseases such as inflammation

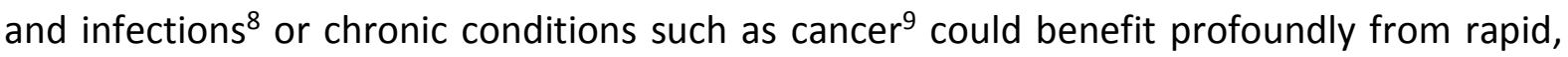


sensitive and cost-effective on-site diagnostic tools. Conventional laboratory techniques, such as enzyme-linked immunosorbent assay (ELISA) ${ }^{10}$ and fluorescent labelling ${ }^{11,12}$ can achieve relevant sensitivity levels of molecular detection, however they require complex optical readout, multiple incubation-washing steps and fluorescent tags that can be expensive, unstable and difficult to produce.

Nanophotonic resonators can efficiently funnel light into nano-scale volumes compatible with biomolecule dimensions, thereby strongly enhancing light-matter interactions and transducing molecular surface binding events into far-field optical signals. ${ }^{13}$ The high sensitivity of nanophotonic biosensors combined with the scalability of detection schemes, low-cost operation and capability for multiplexed measurements make them prominent for biosensing applications. Among the recent ones, optical nanobiosensors based on whispering gallery mode ${ }^{14,15}$ and hyperbolic metamaterials ${ }^{16}$ were shown to provide some of the highest sensitivities down to single-molecule detection. ${ }^{17}$ However, these techniques require complex and bulky read-out set-ups, intricate fabrication procedures and have limited scalability hindering their use as cost-effective and multiplexed biosensors.

On the other hand, nanoplasmonic resonators, made of noble metals, couple the photon energy to the collective oscillations of the metal's free electrons, also known as surface plasmons, which interact with the biomolecules at the metal surface. ${ }^{18} \mathrm{~A}$ number of nanoplasmonic devices have been engineered to efficiently probe the local refractive index variations upon the biomolecular surface binding events yielding high refractometric sensitivities. ${ }^{16,19-23}$ So far, both the propagating $(\mathrm{SPR})^{24}$ and localized surface plasmons $(\text { LSPR })^{25,26}$ have been widely explored for biosensing applications. Particularly, the gold nanohole arrays (Au-NHA) have been a prominent plasmonic system because they can be operated in a robust collinear optical configuration allowing integration in low-cost, easy-touse, and portable platforms. ${ }^{27-30}$ The periodic Au-NHAs exhibit a sharp extraordinary optical transmission (EOT) resonance associated with a dip and a peak in the far-field spectrum due to the interplay of coherently interfering resonant interactions and grating effects (Fig. 1E i.). ${ }^{31}$ Previously, Au-NHAs were explored for numerous biosensing applications ranging from the detection of proteins ${ }^{32,33}$ to exosomes, ${ }^{34}$ viruses, $^{35,36}$ bacteria $^{37}$ and cell secretion, ${ }^{38}$ mainly based on spectral shift monitoring. In an attempt to miniaturize the Au-NHA based biosensors, intensity imaging in transmission was used to detect the shifts of the EOT peak without the need of a spectrometer. ${ }^{27-29}$ Intensity imaging enables 2D large-area detection 
and can be performed using a narrowband illumination source, such as an LED, tuned to the EOT peak to monitor variations in the transmittance due to the spectral shift of the resonance (see Figure $1 \mathrm{E}$ ii.). Although the approach can be realized with a 2D image sensor array, such as charge-coupled device (CCD) or complementary metal-oxide-semiconductor (CMOS), on a portable optical reader, its previous implementation ${ }^{28}$ using Au-NHAs could only achieve detection of $4 \mu \mathrm{g} / \mathrm{ml}$ protein, significantly above the relevant clinical concentrations of most biomarkers. In these conventional spectroscopic and imaging read-out schemes, the signal transduction relies on the spectral shifts in plasmonic transmission resonances due to refractive index changes induced by the accumulation of the analytes on the sensor surface with a certain layer density and thickness. Consequently, the sensitivity levels of such approaches are inherently limited by the concentration and the size of the analyte molecules, as well as non-specific interactions from complex samples.

Here, we present a nanoparticle (NP) enhanced imaging-based plasmonic biosensing technique using Au-NHAs that enables highly sensitive protein detection with single analyte resolution. By digital quantification and localization of individual Au-NPs (100 nm diameter) under bright-field imaging on large area plasmonic imaging surface, the technique enables the detection of single nanoparticle-labeled proteins. We implement the technique in a onestep sandwich immunoassay. After being captured by the first (capture) antibody immobilized on plasmonic Au-NHAs, the protein biomarker is recognized by a second (detection) antibody conjugated to Au-NPs in solution (Figure 1A). Upon binding to the analyte, Au-NPs locally disturb the near-fields of the Au-NHAs (Figure 1E iii.), leading to a strong local transmission suppression in the far-field. These distortions in the transmission from the small vicinity of the NPs are detected under narrow-band illumination at the EOT peak in the visible range (Figure 1B-C), creating plasmonic intensity heatmaps that allow visualization of individual nanoparticle-labeled analytes as high contrast spots. This digital detection approach is particularly distinct from plasmonic detection mechanisms that use NPs to amplify the magnitude of the resonance peak shift in conventional refractive index change-based sensors operating in ensemble-averaged read-out. ${ }^{39-41}$

We first applied this biosensing approach in a proof-of-principle detection of biotinylated bovine serum albumin (bBSA, $67 \mathrm{kDa}$ ) and then human C-reactive protein (CRP, $100 \mathrm{kDa}$ ), a well-established biomarker used for clinical diagnosis and management of acute inflammatory diseases. ${ }^{39-42}$ We show that our approach enables the detection of bBSA down 
to $10 \mathrm{pg} / \mathrm{ml}$ and CRP down to $27 \mathrm{pg} / \mathrm{ml}$ limit-of-detection (LOD). This result is at least four orders of magnitude below CRP levels determining patients at high risk of inflammatory diseases ${ }^{43,44}$ and is well comparable to the established fluorescence amplification techniques, such as ELISA. ${ }^{45}$ At the same time, our technique avoids lengthy signal amplification steps and the plasmonic chips and NPs are stable over time, unlike fluorescence labels that experience photobleaching. In addition, the plasmonic signals are highly confined at the sensor interface and are unaffected by the bulk background, which enables the method to be used in realtime measurements. Importantly, the Au-NHA sensors are fabricated using low-cost waferscale deep-UV lithography (DUVL) producing exceedingly robust and uniform large-area chips on solid transparent substrates (Figure 1D). ${ }^{46}$ In combination with a simple bright-field imaging set-up in a microarray format, our biosensing technology can be scaled to perform clinically relevant biomarker detection in a highly multiplexed manner.

\section{RESULTS AND DISCUSION Principle of detection}

The extraordinary optical transmission in Au-NHAs occurs through the hybridization of propagating surface plasmon polaritons (SPP) and localized surface plasmons (LSP) coupled to the nanoholes. ${ }^{31}$ The propagating SPPs are excited by normally incident light through momentum matching by the periodic nanohole grid, due to a phenomenon known as Wood's anomaly. ${ }^{47}$ This subradiant propagating mode is strongly damped through the radiation channels created by the strong field localizations at the nanoholes, leading to sharp Fanoshaped transmission peaks at the resonant wavelengths in the far-field spectrum (Figure 1E i.). The transmission peak position is highly sensitive to the refractive index changes at the gold-medium interface and therefore can be used for the detection of molecular binding at the NHA surface. ${ }^{48}$

Contrary to the conventional detection approaches based on spectral peak shift monitoring, 27-29,32-38 our transduction relies on the far-field intensity-imaging of the local EOT distortion induced by the binding of functionalized nanoparticles. To numerically investigate the local effects created by the Au-NP and the associated changes in the far-field spectrum of the AuNHAs, we used a commercially available finite-element frequency domain solver (CST microwave studio 2016, Materials and Methods Section). A unit-cell, composed of $3 \times 3 \mathrm{Au}$ 
nanohole array (diameter $=200 \mathrm{~nm}$ period $=600 \mathrm{~nm}$ ) with periodic boundary conditions, is excited from top with normally incident TM mode (Figure $2 A$ ). The size of the simulated $3 \times 3$ unit cell was chosen to match to the diffraction-limited spot $(1.8 \mu \mathrm{m})$ resolved in our experimental imaging set-up.

When the $100 \mathrm{~nm}$ diameter gold nanoparticle binds to the inner walls or the vicinity of the nanohole rim, the localized dipolar resonance is disturbed. This local distortion in the resonant interactions leads to alterations in the radiation pathways and creates suppression of the EOT peak in the far-field (Figure 2B). We explored the effect of the NP position on the resonance modes and found that when a NP binds to the top surface of Au-NHA, its impact on the transmission depends on how close the NP is to the nanohole rim, where the hot-spots of localized nanohole modes reside. Figure 2B shows that as the NP gets closer to the nanohole, the peak suppression increases, which correlates with the distortion of the localized enhanced fields presented in Figure 2C. When a NP is on the gold surface far from the nanohole, we observed that its effect on the NHA transmission is insignificant. This could be due to the spatially limited interaction of the propagating plasmon modes with the small NP volume. Whereas, a NP inside a nanohole produces a drastic distortion on the localized modes, suppressing the transmission significantly. Since the Au-NHAs have a symmetric geometry, their resonances are polarization independent and can be excited with unpolarised light. A NP trapped inside the nanohole can distort the dipolar modes in all lateral directions (see Figure S1. for excitation polarization effect), resulting in a stronger suppression than the surface bound NPs close to the nanohole rim. Importantly, these numerical results are in agreement with the measured NHA transmission spectra and the NP suppression (see Figure S3).

We also investigated the effects of NP size on the numerically computed far-field transmission spectra when Au-NPs of various diameters are entrapped inside a nanohole. Figure 2D shows that the suppression of the transmission peak increases with the NP diameter and saturates at $100 \mathrm{~nm}$. Moreover, it is essential that experimentally the NPs can easily fit inside the nanoholes, both of which can have minor size variations. This defined our choice in the experimental measurements of Au-NPs with $100 \mathrm{~nm}$ average size ( $\pm 10 \%$ dispersity).

We also numerically investigated the effect of a dielectric $\mathrm{SiO}_{2} \mathrm{NP}$ with $110 \mathrm{~nm}$ diameter and did not observe any amplitude change in the transmission peak of the Au-NHA, whether placed inside or outside of the nanohole (Figure S2). These are expected outcomes as the 
local fields can penetrate through the dielectric media without being distorted, yet a minuscule shift in the resonance wavelength is caused by the change in the effective refractive index of the top medium.

The strong local transmission quenching induced by the gold nanoparticle is critical for our distinctive detection mechanism. Numerical calculations indicate that individual particles can produce sufficiently strong spikes on the captured intensity contrast images from the plasmonic surface to be detected in simple bright-field scheme.

\section{Plasmonic imaging of Au NPs on Au-NHAs}

For experimental demonstrations we used uniformly patterned Au-NHA (diameter $=200 \mathrm{~nm}$, period $=600 \mathrm{~nm}$ ) sensor chips of area $1 \mathrm{~cm}^{2}$. The plasmonic nanostructures uniformly covering entire 4-inch quartz wafers were fabricated using a robust high-throughput DUVL lithography ${ }^{46}$ (Figure 1D), yielding multiple low-cost sensor chips (50 chips/wafer), which is crucial for biosensing applications.

We post-patterned Au-NHA wafers defining $10 \times 10$ microarray regions on each chip by patterning labels using photolithography and metal (Ti) lift-off techniques (Figure 1D). The size of each labelled microarray region is $800 \mu \mathrm{m} \times 800 \mu \mathrm{m}$ and each microarray region can be functionalized with $2 \times 2$ bioreceptor sensing spots (150 $\mu$ m diameter spot), providing a flexible platform for multiplexed measurements.

Our experiments are performed on an inverted microscope with a narrow-band illumination, centred at $650 \mathrm{~nm}$ with $10 \mathrm{~nm}$ full width at half maximum (FWHM), where the images are recorded at 1 s exposure on a $1608 \times 1608$ pixels grayscale CMOS camera. The optical path is configured to reach $30 x$ total magnification using a 0.3 numerical aperture (NA) objective lens and a 0.13 NA condenser. This optical arrangement enables the capture of images covering a $385 \times 385 \mu \mathrm{m}^{2}$ area, with a Rayleigh diffraction-limited spot size of

$$
R=1.22 \frac{\lambda_{\text {illumination }}}{N A_{\text {condenser }}+N A_{\text {objective }}} \sim 1.8 \mu \mathrm{m},
$$

Note that this diffraction-limited area corresponds to a $3 \times 3$ nanohole unit cell, which is considered in the numerical analysis above. A detailed description of the optical set-up can be found in Materials and Methods.

To accurately extract the positions of NPs from the imaging data, we acquire the images of bare Au-NHA chips patterned with non-transparent Ti alignment marks prior to the bioassay 
(Figure 3A). This is important because the transmission of bare Au-NHAs exhibits minor spatial intensity variations, independent of the imaging set-up. This could be attributed to several reasons, such as minor differences in the metal film crystal structure or the nanohole uniformity. In order to account for these minor intensity variations, we reference the images of Au-NHA sensors before and after the bioassay with NPs.

Next, we perform the sandwich bioassay by first functionalizing the Au-NHA chips using a noncontact micro-dispenser with 2D arrays of capture molecules through gold-thiol surface chemistry. Then, the chips are successively incubated with the analyte dilutions and a suspension of Au-NPs conjugated to receptor molecules. See Materials and Methods section for details. After the sandwich assay, the Au-NHA chips are rinsed, dried and imaged again (Figure $3 \mathrm{~B}$ ). The images of the corresponding microarray regions before and after capturing the target analyte and NPs are aligned and subtracted using a custom-made Matlab function. The two images are first matched by referencing to identical microarray alignment marks, then scaled to the same average intensity range, corrected for background illumination gradients, subtracted and normalized (see Materials and Methods for details). The alignment and subtraction of the images before and after yield highly accurate heatmaps of NPs binding on Au-NHAs, represented as local intensity spikes (Figure 3C). Finally, the NPs are automatically identified and quantified from the spikes on the plasmonic heatmap with a fixed-value threshold. The optimal threshold depends on the imaging characteristics of the optical set-up, and was empirically found to be $5 \%$ intensity change in our microscope system by mapping plasmonic images to high-resolution Scanning Electron Micrographs (SEM) for NP verification. The optimal threshold value was chosen to detect the maximum number of nanoparticles, while keeping a low rate of false positive signals (see Figure S4 and Figure S5 for details).

\section{SEM validation of plasmonic imaging}

To quantitatively analyze the accuracy of our NP enhanced plasmonic imaging technique we matched plasmonic images to the SEMs of the microarray elements. Figure 3D shows two representative plasmonic heatmap areas and their corresponding SEMs from NP-deposited microspot (I) and NP-free region (II). The details on the test sample preparation used for the system characterization are provided in the Materials and Methods. 
In agreement with the simulations, most of the detected NPs are bound inside the nanoholes, which are marked with red circles on the SEMs in Fig 3D (also shown in Fig.3E-1). Intensity peaks, recognised as NPs, are highlighted by red stars on the plasmonic images. A small fraction of NPs outside of nanoholes, close to the edges of the holes, could also be detected, supporting our numerical results regarding NP-NHA interactions through disruption of localized nanohole modes (Fig.3E-3). As expected, the NPs that are bound far from the nanoholes were not detected (Fig.3E-2). After mapping over 1000 NPs on numerous SEM images, we found that $40 \%$ of NPs bind inside nanoholes and $60 \%$ over the gold surface as summarized in Figure 3F. Interestingly, the areal coverage of the hole area is only $9 \%$, while $40 \%$ of particles bind inside the holes, suggesting that diffusing NPs preferably bind inside the cavities of nanoholes. ${ }^{49}$ From the plasmonic heatmaps, we are able to detect approximately $40 \%$ of all nanoparticles, with a $3 \%$ false positive signal rate based on data with a sample size of 127 plasmonic intensity peaks matched to SEM. In addition, our detection is robust against non-specifically binding agglomerates of NPs and large sedimenting contaminants, since they can be easily identified and discarded by the size and the shape of intensity spikes on the plasmonic imaging surface (see Figure S6).

\section{Sandwich assay protein detection}

We first show the biosensing potential of our NP-enhanced plasmonic imaging method by detecting bBSA as depicted in Figure 4A. We start by acquiring the reference images on the bare chips that will be used for the bioassays. Next, the Au-NHAs were surface functionalized with PEG-thiol chemistry to prevent non-specific fouling and ensure optimal protein immobilization through EDC-NHS crosslinker. Then, microarrays of neutravidin (150 pl droplets spotted with $200 \mu \mathrm{m}$ pitch) were formed using a non-contact micro-dispenser and the remaining areas were blocked with BSA to deactivate non-reacted crosslinker groups. The bBSA dilutions were spiked in $100 \mu \mathrm{PBS} 1 \mathrm{x}$ and incubated with the Au-NHA chips. Finally, a suspension of Au-NPs covalently tethered to streptavidin in PBS 1x with $1 \%$ BSA was incubated. After rinsing with milliQ water and drying under $\mathrm{N}_{2}$ stream, the second set of images was acquired. Further details on the bioassay procedure are given in the Materials and Methods section. To quantify bBSA, the image datasets were processed as described above. The local intensity spikes were counted over $100 \times 100 \mu \mathrm{m}^{2}$ neutravidin spotted areas (see Fig. 4B), and the results are correlated to the bBSA concentrations (Fig. 4C). We 
experimentally show the successful detection of bBSA concentrations down to $10 \mathrm{pg} / \mathrm{ml}$, which corresponds to our estimated limit-of-detection (LOD, computed by adding three times the standard deviation of control signal to the average of the control). These results show that our method reaches the sensitivity of fluorescence amplification techniques, while avoiding the signal amplification step, which is used in a typical ELISA. To put into context, a concentration of $10 \mathrm{pg} / \mathrm{ml}$ of bBSA translates to $9 \times 10^{6}$ molecules in a $100 \mu \mathrm{l}$ sample incubated on a sensor chip $\left(1 \times 1 \mathrm{~cm}^{2}\right)$. The ratio of a single sensing spot $\left(100 \times 100 \mu \mathrm{m}^{2}\right)$ to the entire sensor chip $\left(1 \times 1 \mathrm{~cm}^{2}\right)$ is roughly 1:104. Accounting for molecular diffusion limitation, several hundred molecules reach to a single sensing spot of $100 \times 100 \mu \mathrm{m}^{2}$. Taking into account the diffusion limitation of NPs and that $40 \%$ of bound particles can be detected (inside or close to the nanoholes), the $10 \mathrm{pg} / \mathrm{ml}$ concentration would result in approximately $\sim 10-100 \mathrm{NP}$ counts per sensing spot. This estimation is in agreement with our assay, where the $10 \mathrm{pg} / \mathrm{ml}$ bBSA concentration yields on average 50 NP counts per read-out spot. The above results indicate that our detection method ultimately operates near the actual diffusion limitation of the system. In this way, the extraction of intensity spikes from the plasmonic imaging heatmap allows us to digitally count the analytes with high sensitivity, contrary to conventional affinity sensors where the signals are averaged over sensor areas. Moreover, this sensitivity limit could be further improved by addressing the diffusion limitation with active transport mechanisms. ${ }^{50}$

The flexibility of our method enables its use in a variety of bioanalytical applications, by using appropriate biofunctionalization procedures. In order to show the diagnostics potential of the platform, we detect human C-reactive protein (CRP). Human CRP is a blood biomarker, whose levels elevate during acute inflammatory conditions, such as sepsis and coronary heart disease. Septic shock is caused by the response of body to infections and is one of the most pressing healthcare challenges worldwide with millions of patients diagnosed each year and over $20 \%$ of lethal incidence. ${ }^{51}$ Therefore, the development of biosensors enabling timely and accurate detection of biomarkers such as CRP can have a profound impact on the effective disease management.

We use the proposed method in a single-step sandwich immunoassay to detect human CRP from bovine serum albumin (BSA) solution. Fig.4D illustrates the CRP detection and the details of the assay can be found in the Materials and Methods section. 
We experimentally detect CRP concentrations down to $27 \mathrm{pg} / \mathrm{ml}$, which is four orders of magnitude within clinically critical limit, thus opening the path for early-stage disease monitoring. As expected, the LOD for CRP is slightly higher than $10 \mathrm{pg} / \mathrm{ml}$ LOD in bBSA detection (Figure $4 \mathrm{E}, \mathrm{F}$ ), since the CRP assay was performed in media with high BSA concentration, which may screen antibody-antigen interactions. Additionally, the affinity between anti-CRP antibodies and CRP are normally lower than the affinity between biotin and streptavin or neutravidin.

Figure 4 C,F presents the calibration curves of bBSA and CRP measurements. The non-specific nanoparticle adsorption, indicated with dashed grey lines, was the main limitation for the LOD in both assays. The control experiments were performed by incubating chips in samples containing no analyte (i.e. bBSA or CRP), while all other assay steps remained unchanged. In CRP measurements, the NP concentration was higher than in bBSA assay, which was reflected on higher specific NP counts, but also higher baseline signal from non-specific NP adsorption. Importantly, our current results are well comparable to the high performance of commercial ELISA kits, which can detect down to $3-15 \mathrm{pg} / \mathrm{ml}$ of human CRP when performed in a fully equipped clinical laboratories by trained personnel. ${ }^{42}$

To show a proof-of-principle operation in complex samples, we perform detection of human CRP spiked in supplemented cell culture media with $10 \%$ horse serum (see Figure S7 for results). We confidently detect down to $100 \mathrm{pg} / \mathrm{ml}$ of human CRP spiked in supplemented media, and the calculated limit of detection (LOD) is $69 \mathrm{pg} / \mathrm{ml}$. The dynamic linear range lies between $500 \mathrm{pg} / \mathrm{ml}$ and approximately $10 \mathrm{ng} / \mathrm{ml}$, which is well within the clinically relevant values. In order to further optimize the assay for different concentration ranges of different biomarkers, the dynamic range of measurement could be adjusted, for example by changing the concentration of NPs. Moreover, our technique can be used in real-time measurements in aqueous environment as well (Figure S8 and supporting video show NPs binding to NHA inflow), because the localized field disruption of Au-NHAs by Au-NPs is highly surface-confined and is also valid for the plasmonic resonances in wet medium.

Further development of the assay biochemistry and applications with serum or whole blood samples, as well as multiplexed biomarker detection will be essential to explore the full potential of our approach. Implementation of a compact optical reader, which essentially comprises a narrow-band illumination source, an optical path generating 1-2 $\mu \mathrm{m}$ spatial 
resolution and a CMOS sensor in conjunction with image processing algorithms, will be another step to adapt the technology for on-site diagnostic applications.

\section{CONCLUSIONS}

In conclusion, we present a NP-enhanced imaging-based plasmonic biosensing technique using large-area Au-NHA sensors. We show that by disrupting localized plasmon modes in AuNHA surface with Au-NPs, our method produces plasmonic heatmaps that visualize single sub-wavelength Au-NPs under bright-field imaging. Consequently, by detecting individual AuNP-labeled molecules we can digitally quantify the single analyte binding events on the imaging surface, enabling multiplexed detection of biomarkers at low concentrations, eminently at the level of current gold-standard laboratory methods, such as ELISA. At the same time, our technique avoids multi-step staining procedures and does not rely on advanced read-out set-ups, such as required in fluorescent techniques. We implemented the proposed method in sandwich immunoassay measurements of bBSA and human CRP, achieving clinically relevant detection limits of 10 and $27 \mathrm{pg} / \mathrm{ml}$ from buffer, respectively, and $69 \mathrm{pg} / \mathrm{ml}$ of human CRP from supplemented cell media. Prominently, the large-area Au-NHA sensor chips are fabricated in a large-scale low-cost manufacturing, enabling high-throughput biosensing applications. Overall, our simple and scalable detection approach paired with robust sensor chips presents a flexible platform for highly sensitive multiplexed measurements in various settings, including for point-of-care applications.

\section{MATERIALS AND METHODS}

Gold nanohole array Eminently, the fabrication of Au-NHAs was tion

Gold nanohole arrays (Au-NHAs) were fabricated using high-throughput wafer-scale deep-UV lithography (DUVL) and ion beam etching techniques. First, 4-inch fused silica wafers were cleaned with RCA solution (1:1:5 $\left.\mathrm{H}_{2} \mathrm{O}_{2}: \mathrm{NH}_{4} \mathrm{OH}: \mathrm{H}_{2} \mathrm{O}\right)$, rinsed with deionized water and dried under nitrogen stream. Cleaned wafers were coated with $10 \mathrm{~nm}$ of titanium (Ti) and 120 $\mathrm{nm}$ of gold $\mathrm{Au}$ ) in Alliance-Concept EVA 760 electron-gun evaporator. The NHAs of $600 \mathrm{~nm}$ period and $200 \mathrm{~nm}$ diameter were patterned using a $248 \mathrm{~nm}$ deep-UV stepper (ASML PAS $5500 / 300$ DUV). The nanohole arrays were transferred into metal films with ion-beam etching (Oxford Instruments PlasmaLab $300 \mathrm{IBE}$ ) and the photoresist was stripped with oxygen plasma.

Microarray marks on Au-NHAs were formed with Ti to enable image recognition and alignment. The NHA wafers were coated with $1.3 \mu \mathrm{m}$ AZ1512 positive photoresist and microarray patterns were exposed using Heidelberg MLA150 laser writer. After resist 
development, $50 \mathrm{~nm}$ Ti was evaporated using an electron gun evaporator. Next, the wafers were diced into $1 \times 1 \mathrm{~cm}^{2}$ chips. The resist was removed from chips by immersing in resist remover with sonication at $70^{\circ} \mathrm{C}$ for 2 hours. Finally, the chips were cleaned in oxygen plasma for 5 min at $500 \mathrm{~W}$ and RCA solution (1:1:5 $\mathrm{H}_{2} \mathrm{O}_{2}: \mathrm{NH}_{4} \mathrm{OH}: \mathrm{H}_{2} \mathrm{O}$ by volume) for 1 min to ensure uniformly clean gold surface.

\section{Numerical simulations}

To numerically investigate the local field effects on Au-NHAs created by the Au-NPs and the associated changes in the far-field spectrum, we used a commercially available finiteelement frequency domain (FEFD) solver (CST microwave studio 2016, Materials and Methods Section). We simulated Au-NHAs of $200 \mathrm{~nm}$ diameter and $600 \mathrm{~nm}$ period in an Au / $\mathrm{Ti}(120 \mathrm{~nm} / 10 \mathrm{~nm}$ ) thin film (optical parameters from Johnson and Christy) on a $500 \mathrm{~nm}$ thick $\mathrm{SiO} 2$ substrate with refractive index $(\mathrm{RI})=1.46$ and air background media $(\mathrm{RI}=1)$. The Ti layer not only serves for Au adhesion to the silica substrate in fabrication, but also suppresses undesired gold-substrate modes, ensuring sharp shape and good isolation of plasmonic resonant modes in Au-NHA transmission. ${ }^{36,52}$ The simulated unit cell contains $3 \times 3$ nanoholes with periodic boundary conditions. The unit cell size was chosen to correspond to the $\sim 1.8$ $\mu \mathrm{m}$ diffraction limited spot resolved in our experiments. The illumination is set from top with normally incident TM mode. The electric field was monitored at $650 \mathrm{~nm}(461 \mathrm{THz})$.

\section{Optical set-up}

The microscope measurements were performed on an inverted Nikon Eclipse Ti-E system. A tungsten halogen lamp was used for illumination together with a narrow-band optical filter (Thorlabs) with $650 \mathrm{~nm}$ center wavelength and $10 \mathrm{~nm}$ full width at half maximum, matching the plasmonic resonance of Au-NHAs in dry. Optical path included a 20x and $0.3 \mathrm{NA}$ objective lens and a 1.5x intermediate microscope magnification. Images were recorded using a Nikon Qi2 camera with 1 second exposure and 1.2 digital gain.

Spectral characterization of NHA transmission was performed on Nikon Eclipse Ti-E system. A tungsten halogen lamp was used for broad-band illumination. Spectra were acquired using Andor SR-303i imaging spectrometer and recorded on an Andor iKon-M camera.

\section{Image processing}

The plasmonic image heatmaps are obtained by computing the normalized absolute difference between an image of NHA with NPs and a reference image of bare NHA:

$$
I_{x, y}=\frac{\left|\operatorname{Ire} f_{x, y}-\operatorname{In} p_{x, y}\right|}{\text { mean(Iref) }}
$$

Where $\operatorname{Ire} f_{x, y}$ is the intensity of pixel $[\mathrm{x}, \mathrm{y}]$ on the reference image of bare NHA. In this way, plasmonic heatmap image presents the absolute values of the normalized intensity changes at every pixel position.

NPs binding causes a decrease in the transmitted intensity, however we choose positive sign convention for illustration convenience.

\section{Chemicals and biologicals}

Ammonium hydroxide solution (ACS reagent, 28-30\%), hydrogen peroxide ( $\mathrm{H} 2 \mathrm{O} 230$ $\%), \mathrm{N}$-hydroxysulfosuccinimide sodium salt (sulfo-NHS), N-(3-dimethylamino propyl)- $\mathrm{N}^{\prime}$ ethylcarbodiimide hydrochloride (EDC), 2-(N-Morpholino)ethanesulfonic acid (MES), bovine serum albumin (BSA) lyophilized, biotinylated BSA (bBSA), neutravidin, phosphate buffered saline (PBS), Tween ${ }^{\circledR} 20$ and horse serum were purchased from Sigma-Aldrich. Cell culture medium was from ThermoFisher Scientific. Ethanol (EtOH), absolute was from Thermo 
Chemicals. Polyethylene-glycol (PEG) thiols terminated with hydroxyl (HS-C6-(EG)4-OH) and carboxyl (HS-C11-(EG)4-OCH2-COOH) groups, were purchased from Prochimia. Human Creactive protein (CRP) and anti-CRP monoclonal IgG antibodies PCR-196 and PCR-183, used as capture and recognition antibodies respectively, were provided by Diesse Diagnostica Senese. Spherical gold nanoparticles of $100 \mathrm{~nm}$ dimeter coated with streptavidin or with protein-G, supplied at optical density (OD) 3 in PBS with $20 \%$ glycerol and $1 \%$ BSA, were purchased from Cytodiagnostics.*

*A $100 \mathrm{~nm}$ diameter gold nanoparticles concentration of optical density (OD) 1 corresponds to $5.6 \mathrm{E} 9$ nanoparticles per $1 \mathrm{ml}$ solution; the relation between OD and NP/ml units is linear.

\section{Au-NHA functionalization}

After cleaning in oxygen plasma and RCA, Au-NHA chips were immersed and incubated overnight in $1 \mathrm{mM}$ PEG-thiol solution in anhydrous ethanol with $\mathrm{OH}: \mathrm{COOH}$ terminated PEG mixed in 5:1 ratio. After incubation, chips were gently rinsed with fresh ethanol and milliQ water, then dried under $\mathrm{N}_{2}$ stream. The chips were activated in $70 \mathrm{mg} / \mathrm{ml} \mathrm{EDC}$ and $20 \mathrm{mg} / \mathrm{ml}$ s-NHS mixture in $0.1 \mathrm{M}$ MES buffer for 20 minutes. Next, the chips were rinsed in milliQ water and dried under $\mathrm{N}_{2}$ stream. Monoclonal anti-CRP IgG196 or neutravidin were immediately spotted on the activated Au-NHAs using Scienion S3 piezoelectric non-contact microdispenser. The spotting solution contained either neutravidin or anti-CRP IgG at 200 $\mu \mathrm{g} / \mathrm{ml}$ in PBS $1 \mathrm{x}$ with $0.5 \%$ glycerol. The average droplet volume was $160 \mathrm{pl}$ and the contact area of the formed spot was approximately $100 \mu \mathrm{m}$ in diameter. The spotted chips were incubated overnight in a humid atmosphere at $4^{\circ} \mathrm{C}$ and then blocked with BSA $1 \%$ solution in PBS to passivate the non-reacted EDC-activated groups. Functionalized chips were used in measurements on the same day.

\section{Biotin-BSA sandwich assay}

Au-NHA chips spotted with neutravidin and blocked with BSA were briefly rinsed in PBS and immersed in $100 \mu \mathrm{l}$ of biotin-BSA calibration dilutions prepared in PBS $1 \mathrm{x}$ and incubated for 1 hour. Next, each chip was rinsed in PBS $1 x$ with BSA $1 \%$ and $0.05 \%$ Tween 20 for 20 minutes. After a brief immersion in PBS $1 x$ with BSA $1 \%$, the chips were incubated for 1 hour in Streptavidin-conjugated gold nanoparticles diluted to OD 0.1 in PBS 1x BSA $1 \%$. After incubation, the chips were rinsed in milliQ water for 5 mins and dried under gentle $\mathrm{N}_{2}$ stream.

The fits presented in Figures 4C and 4F were obtained using Michaelis-Menten kinetic model. The error bars were calculated as the standard deviation from the measurements of at least 5 different biosensing areas of at least 2 different technical replicates (different plasmonic NHA chips measured at different days).

\section{Au nanoparticle conjugation to anti-CRP antibodies}

$40 \mu \mathrm{l}$ of $100 \mathrm{~nm}$ gold nanoparticles conjugated to protein $\mathrm{G}$ at OD 3 concentration were mixed with $1 \mu \mathrm{l}$ of $2.1 \mathrm{mg} / \mathrm{ml}$ anti-CRP PCR183 antibody by gentle pipetting and incubated overnight at $4{ }^{\circ} \mathrm{C}$. Next day, the nanoparticle-antibody solution was diluted in $1 \mathrm{ml}$ of PBS with $1 \%$ BSA and centrifuged at $200 \mathrm{~g}$ until a pellet was formed. After removing the supernatant, the pellet was resuspended in $1 \mathrm{ml}$ of PBS $1 \mathrm{x}$ with $1 \%$ BSA. Centrifugation and resuspension steps were performed 4 times to remove unbound antibodies from NPs suspension. After the final centrifugation and supernatant removal, the NPs were resuspended in $40 \mu \mathrm{l}$ of PBS $1 \mathrm{x}$ with $1 \%$ BSA. The conjugated nanoparticles were used for measurements on the same day. 


\section{CRP sandwich assay}

Au-NHA chips spotted with anti-CRP IgG 196 and blocked with BSA were briefly rinsed in PBS and immersed in $100 \mu \mathrm{l}$ of CRP calibration dilutions prepared by spiking CRP in PBS $1 \mathrm{x}$ with $1 \%$ BSA. After 1 hour incubation, chips were rinsed in PBS $1 x$ with BSA $1 \%$ and $0.05 \%$ Tween for 20 min with gentle agitation on a gyro-rocker. After brief immersion in PBS 1x with BSA $1 \%$, the chips were incubated for 1 hour in IgG183-coated Au-NPs diluted to OD 0.2 in PBS $1 x$ BSA $1 \%$. After incubation, chips were rinsed in milliQ water for $5 \mathrm{~min}$ and dried under gentle $\mathrm{N}_{2}$ stream.

\section{ASSOCIATED CONTENT}

\section{Supporting Information}

Supporting Information Available: This material is available free of charge via the Internet at ACS Publications website at DOI: $x x x / x x x$

Additional figures summarize the numerical simulations, spectral characterization, statistical analysis and the results of CRP detection from media (PDF).

\section{AUTHOR INFORMATION \\ Corresponding Author: \\ *E-mail: hatice.altug@epfl.ch \\ Present Address}

EPFL STI IBI-STI BIOS Station 17, BM 4133, 1015 Lausanne, Switzerland

Notes

The authors declare no competing financial interests.

\section{ACKNOWLEDGEMENTS}

We would like to thank Center of MicroNano Technology at École Polytechnique Fédérale de Lausanne for providing support on the nanofabrication; Diesse diagnostic senese SPA for providing human C-reactive protein (CRP) and anti-CRP monoclonal IgG antibodies. We acknowledge the support of the École Polytechnique Fédérale de Lausanne and the European Union's Horizon 2020 research and innovation program under grant agreement no. 644956 (RAIS project). 


\section{REFERENCES}

(1) Fan, X.; White, I. M.; Shopova, S. I.; Zhu, H.; Suter, J. D.; Sun, Y. Sensitive Optical Biosensors for Unlabeled Targets: A Review. Anal. Chim. Acta 2008, 620, 8-26.

(2) Anker, J. N.; Hall, W. P.; Lyandres, O.; Shah, N. C.; Zhao, J.; Van Duyne, R. P. Biosensing with Plasmonic Nanosensors. In Nanoscience And Technology: A Collection of Reviews from Nature Journals; World Scientific, 2010; pp 308-319.

(3) Zeng, S.; Baillargeat, D.; Ho, H.-P.; Yong, K.-T. Nanomaterials Enhanced Surface Plasmon Resonance for Biological and Chemical Sensing Applications. Chem. Soc. Rev. 2014, 43, 3426-3452.

(4) Lopez, G. A.; Estevez, M.-C.; Soler, M.; Lechuga, L. M. Recent Advances in Nanoplasmonic Biosensors: Applications and Lab-on-a-Chip Integration. Nanophotonics 2017, 6, 123-136.

(5) Di Fabrizio, E.; Schlücker, S.; Wenger, J.; Regmi, R.; Rigneault, H.; Calafiore, G.; West, M.; Cabrini, S.; Fleischer, M.; Van Hulst, N. F. Roadmap on Biosensing and Photonics with Advanced Nano-Optical Methods. J. Opt. 2016, 18, 63003.

(6) Špačková, B.; Wrobel, P.; Bocková, M.; Homola, J. Optical Biosensors Based on Plasmonic Nanostructures: A Review. Proc. IEEE 2016, 104, 2380-2408.

(7) Jackman, J. A.; Ferhan, A. R.; Cho, N.-J. Nanoplasmonic Sensors for Biointerfacial Science. Chem. Soc. Rev. 2017, 46, 3615-3660.

(8) Dantham, V. R.; Holler, S.; Kolchenko, V.; Wan, Z.; Arnold, S. Taking Whispering GalleryMode Single Virus Detection and Sizing to the Limit. Appl. Phys. Lett. 2012, 101, 43704.

(9) Kosaka, P. M.; Pini, V.; Ruz, J. J.; González, M. U.; Ramos, D.; Calleja, M.; Tamayo, J.; da Silva, R. A. Detection of Cancer Biomarkers in Serum Using a Hybrid Mechanical and Optoplasmonic Nanosensor. Nat. Nanotechnol. 2014, 9.

(10) Rissin, D. M.; Kan, C. W.; Campbell, T. G.; Howes, S. C.; Fournier, D. R.; Song, L.; Piech, T.; Patel, P. P.; Chang, L.; Rivnak, A. J. Single-Molecule Enzyme-Linked Immunosorbent Assay Detects Serum Proteins at Subfemtomolar Concentrations. Nat. Biotechnol. 2010, 28, 595-599.

(11) Dickson, R. M.; Cubitt, A. B.; Tsien, R. Y.; Moerner, W. E. On/off Blinking and Switching Behaviour of Single Molecules of Green Fluorescent Protein. Nature 1997, 388, 355358.

(12) Jain, A.; Liu, R.; Ramani, B.; Arauz, E.; Ishitsuka, Y.; Ragunathan, K.; Park, J.; Chen, J.; Xiang, Y. K.; Ha, T. Probing Cellular Protein Complexes Using Single-Molecule PullDown. Nature 2011, 473, 484-488.

(13) Bozhevolnyi, S. I.; Volkov, V. S.; Devaux, E.; Laluet, J.-Y.; Ebbesen, T. W. Channel Plasmon Subwavelength Waveguide Components Including Interferometers and Ring Resonators. Nature 2006, 440, 508-511.

(14) Su, J.; Goldberg, A. F.; Stoltz, B. M. Label-Free Detection of Single Nanoparticles and Biological Molecules Using Microtoroid Optical Resonators. Light Sci. Appl. 2016, 5, e16001.

(15) Baaske, M. D.; Foreman, M. R.; Vollmer, F. Single-Molecule Nucleic Acid Interactions Monitored on a Label-Free Microcavity Biosensor Platform. Nat. Nanotechnol. 2014, 9, 933-939. 
(16) Sreekanth, K. V.; Alapan, Y.; ElKabbash, M.; Ilker, E.; Hinczewski, M.; Gurkan, U. A.; De Luca, A.; Strangi, G. Extreme Sensitivity Biosensing Platform Based on Hyperbolic Metamaterials. Nat. Mater. 2016, 15, 621.

(17) Walt, D. R. Optical Methods for Single Molecule Detection and Analysis; ACS Publications, 2012.

(18) Raether, H. Surface Plasmons on Smooth Surfaces. In Surface plasmons on smooth and rough surfaces and on gratings; Springer, 1988; pp 4-39.

(19) Shen, Y.; Zhou, J.; Liu, T.; Tao, Y.; Jiang, R.; Liu, M.; Xiao, G.; Zhu, J.; Zhou, Z.-K.; Wang, $X$. Plasmonic Gold Mushroom Arrays with Refractive Index Sensing Figures of Merit Approaching the Theoretical Limit. Nat. Commun. 2013, 4, 2381.

(20) Zijlstra, P.; Paulo, P. M.; Orrit, M. Optical Detection of Single Non-Absorbing Molecules Using the Surface Plasmon Resonance of a Gold Nanorod. Nat. Nanotechnol. 2012, 7, 379-382.

(21) Lindquist, N. C.; Nagpal, P.; McPeak, K. M.; Norris, D. J.; Oh, S.-H. Engineering Metallic Nanostructures for Plasmonics and Nanophotonics. Rep. Prog. Phys. 2012, 75, 36501.

(22) Kabashin, A. V.; Evans, P.; Pastkovsky, S.; Hendren, W.; Wurtz, G. A.; Atkinson, R.; Pollard, R.; Podolskiy, V. A.; Zayats, A. V. Plasmonic Nanorod Metamaterials for Biosensing. Nat. Mater. 2009, 8, 867-871.

(23) Špačková, B.; Lynn Jr, N. S. S.; Šípová, H.; Slabỳ, J.; Homola, J. A Route to Superior Performance of a Nanoplasmonic Biosensor: Consideration of Both Photonic and Mass Transport Aspects. ACS Photonics 2018.

(24) Homola, J. Surface Plasmon Resonance Sensors for Detection of Chemical and Biological Species. Chem. Rev. 2008, 108, 462-493.

(25) Mazzotta, F.; Johnson, T. W.; Dahlin, A. B.; Shaver, J.; Oh, S.-H.; Höök, F. Influence of the Evanescent Field Decay Length on the Sensitivity of Plasmonic Nanodisks and Nanoholes. Acs Photonics 2015, 2, 256-262.

(26) Unser, S.; Bruzas, I.; He, J.; Sagle, L. Localized Surface Plasmon Resonance Biosensing: Current Challenges and Approaches. Sensors 2015, 15, 15684-15716.

(27) Coskun, A. F.; Cetin, A. E.; Galarreta, B. C.; Alvarez, D. A.; Altug, H.; Ozcan, A. Lensfree Optofluidic Plasmonic Sensor for Real-Time and Label-Free Monitoring of Molecular Binding Events over a Wide Field-of-View. Sci. Rep. 2014, 4, 6789.

(28) Cetin, A. E.; Coskun, A. F.; Galarreta, B. C.; Huang, M.; Herman, D.; Ozcan, A.; Altug, H. Handheld High-Throughput Plasmonic Biosensor Using Computational on-Chip Imaging. Light Sci. Appl. 2014, 3, e122.

(29) Ballard, Z. S.; Shir, D.; Bhardwaj, A.; Bazargan, S.; Sathianathan, S.; Ozcan, A. Computational Sensing Using Low-Cost and Mobile Plasmonic Readers Designed by Machine Learning. ACS Nano 2017, 11, 2266-2274.

(30) Najiminaini, M.; Vasefi, F.; Kaminska, B.; Carson, J. J. Nanohole-Array-Based Device for 2D Snapshot Multispectral Imaging. Sci. Rep. 2013, 3.

(31) Ebbesen, T. W.; Lezec, H. J.; Ghaemi, H. F.; Thio, T.; Wolff, P. A. Extraordinary Optical Transmission through Sub-Wavelength Hole Arrays. Nature 1998, 391, 667-669.

(32) Couture, M.; Ray, K. K.; Poirier-Richard, H.-P.; Crofton, A.; Masson, J.-F. 96-Well Plasmonic Sensing with Nanohole Arrays. ACS Sens. 2016, 1, 287-294.

(33) Sharpe, J. C.; Mitchell, J. S.; Lin, L.; Sedoglavich, N.; Blaikie, R. J. Gold Nanohole Array Substrates as Immunobiosensors. Anal. Chem. 2008, 80, 2244-2249. 
(34) Im, H.; Shao, H.; Park, Y. I.; Peterson, V. M.; Castro, C. M.; Weissleder, R.; Lee, H. LabelFree Detection and Molecular Profiling of Exosomes with a Nano-Plasmonic Sensor. Nat. Biotechnol. 2014, 32, 490-495.

(35) Yanik, A. A.; Huang, M.; Kamohara, O.; Artar, A.; Geisbert, T. W.; Connor, J. H.; Altug, H. An Optofluidic Nanoplasmonic Biosensor for Direct Detection of Live Viruses from Biological Media. Nano Lett. 2010, 10, 4962-4969.

(36) Jackman, J. A.; Linardy, E.; Yoo, D.; Seo, J.; Ng, W. B.; Klemme, D. J.; Wittenberg, N. J.; Oh, S.-H.; Cho, N.-J. Plasmonic Nanohole Sensor for Capturing Single Virus-Like Particles toward Virucidal Drug Evaluation. Small 2016, 12, 1159-1166.

(37) Soler, M.; Belushkin, A.; Cavallini, A.; Kebbi-Beghdadi, C.; Greub, G.; Altug, H. Multiplexed Nanoplasmonic Biosensor for One-Step Simultaneous Detection of Chlamydia Trachomatis and Neisseria Gonorrhoeae in Urine. Biosens. Bioelectron. 2017, 94, 560-567.

(38) Li, X.; Soler, M.; Özdemir, C. I.; Belushkin, A.; Yesilkoy, F.; Altug, H. Plasmonic Nanohole Array Biosensor for Label-Free and Real-Time Analysis of Live Cell Secretion. Lab. Chip 2017.

(39) Black, S.; Kushner, I.; Samols, D. C-Reactive Protein. J. Biol. Chem. 2004, 279, 4848748490.

(40) Pradhan, A. D.; Manson, J. E.; Rifai, N.; Buring, J. E.; Ridker, P. M. C-Reactive Protein, Interleukin 6, and Risk of Developing Type 2 Diabetes Mellitus. Jama 2001, 286, 327334.

(41) Simon, L.; Gauvin, F.; Amre, D. K.; Saint-Louis, P.; Lacroix, J. Serum Procalcitonin and CReactive Protein Levels as Markers of Bacterial Infection: A Systematic Review and Meta-Analysis. Clin. Infect. Dis. 2004, 39, 206-217.

(42) Ridker, P. M.; Danielson, E.; Fonseca, F. A.; Genest, J.; Gotto Jr, A. M.; Kastelein, J. J.; Koenig, W.; Libby, P.; Lorenzatti, A. J.; MacFadyen, J. G. Rosuvastatin to Prevent Vascular Events in Men and Women with Elevated C-Reactive Protein. N. Engl. J. Med. 2008, 359, 2195.

(43) Ridker, P. M.; Cannon, C. P.; Morrow, D.; Rifai, N.; Rose, L. M.; McCabe, C. H.; Pfeffer, M. A.; Braunwald, E. C-Reactive Protein Levels and Outcomes after Statin Therapy. $N$. Engl. J. Med. 2005, 352, 20-28.

(44) Harris, T. B.; Ferrucci, L.; Tracy, R. P.; Corti, M. C.; Wacholder, S.; Ettinger, W. H.; Heimovitz, H.; Cohen, H. J.; Wallace, R. Associations of Elevated Interleukin-6 and CReactive Protein Levels with Mortality in the Elderly. Am. J. Med. 1999, 106, 506-512.

(45) Niu, R.; Liu, Y.; Zhang, Y.; Zhang, Y.; Wang, H.; Wang, Y.; Wang, W.; Li, X. iTRAQ-Based Proteomics Reveals Novel Biomarkers for Idiopathic Pulmonary Fibrosis. PloS One 2017, 12, e0170741.

(46) F Yesilkoy; R Terborg; J Pello; A Belushkin; Y Jahani; V Pruneri; H Altug. Phase-Sensitive Plasmonic Biosensor Using a Portable and Large Field of View Interferometric Microarray Imager. Light Sci. Appl. 2018.

(47) Wood, R. W. On a Remarkable Case of Uneven Distribution of Light in a Diffraction Grating Spectrum (from Philosophical Magazine 1902). SPIE Milest. Ser. MS 1993, 83, 287-287.

(48) Brolo, A. G.; Gordon, R.; Leathem, B.; Kavanagh, K. L. Surface Plasmon Sensor Based on the Enhanced Light Transmission through Arrays of Nanoholes in Gold Films. Langmuir 2004, 20, 4813-4815. 
(49) Krishnan, M. Electrostatic Free Energy for a Confined Nanoscale Object in a Fluid. J. Chem. Phys. 2013, 138, 114906.

(50) Shiddiky, M. J. A.; Vaidyanathan, R.; Rauf, S.; Tay, Z.; Trau, M. Molecular Nanoshearing: An Innovative Approach to Shear off Molecules with AC-Induced Nanoscopic Fluid Flow. Sci. Rep. 2014, 4.

(51) Dellinger, R. P.; Levy, M. M.; Rhodes, A.; Annane, D.; Gerlach, H.; Opal, S. M.; Sevransky, J. E.; Sprung, C. L.; Douglas, I. S.; Jaeschke, R. Surviving Sepsis Campaign: International Guidelines for Management of Severe Sepsis and Septic Shock, 2012. Intensive Care Med. 2013, 39, 165-228.

(52) Cetin, A. E.; Etezadi, D.; Galarreta, B. C.; Busson, M. P.; Eksioglu, Y.; Altug, H. Plasmonic Nanohole Arrays on a Robust Hybrid Substrate for Highly Sensitive Label-Free Biosensing. ACS Photonics 2015, 2, 1167-1174. 

Figures
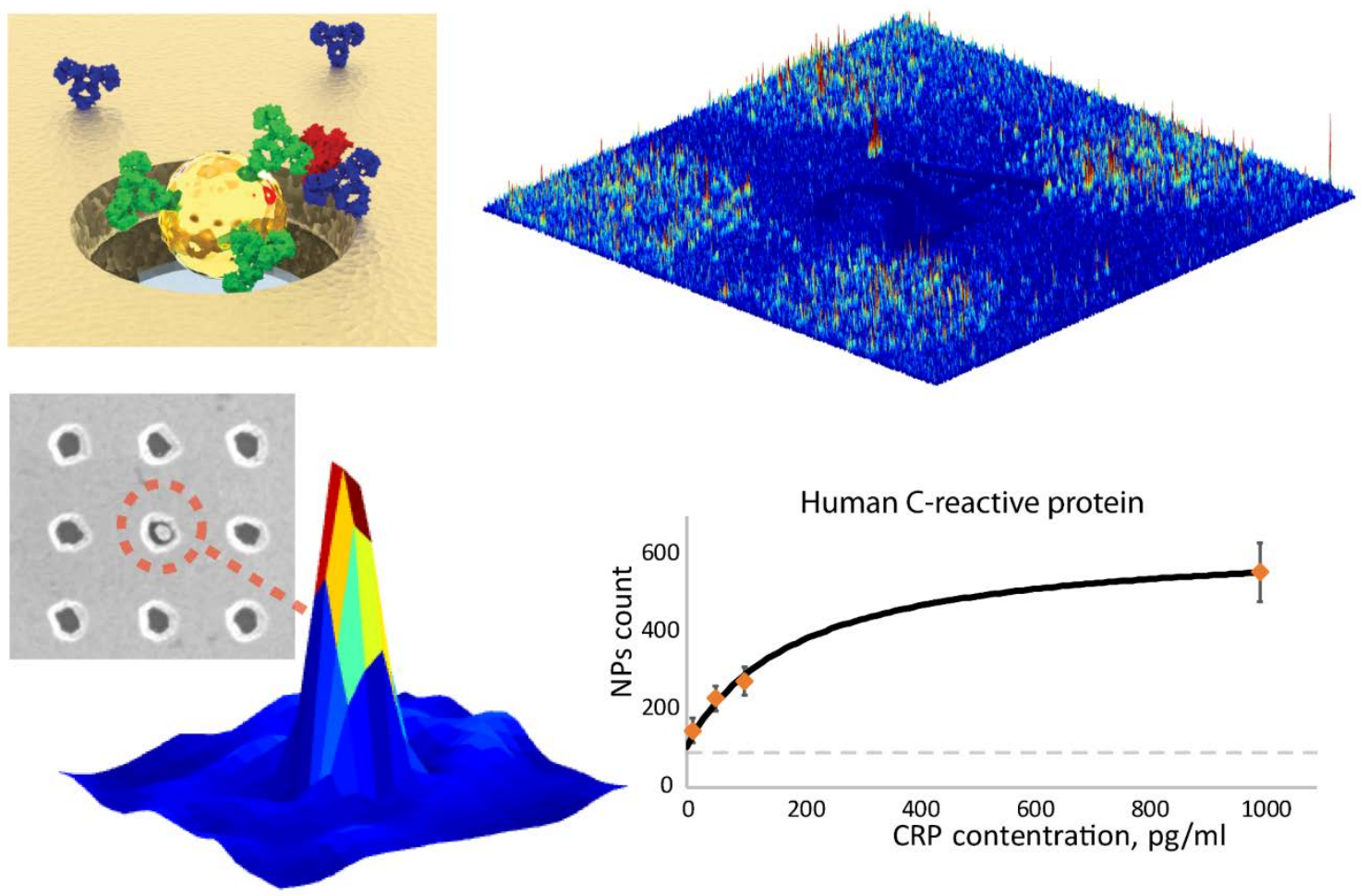

Scheme 1 Abstract 

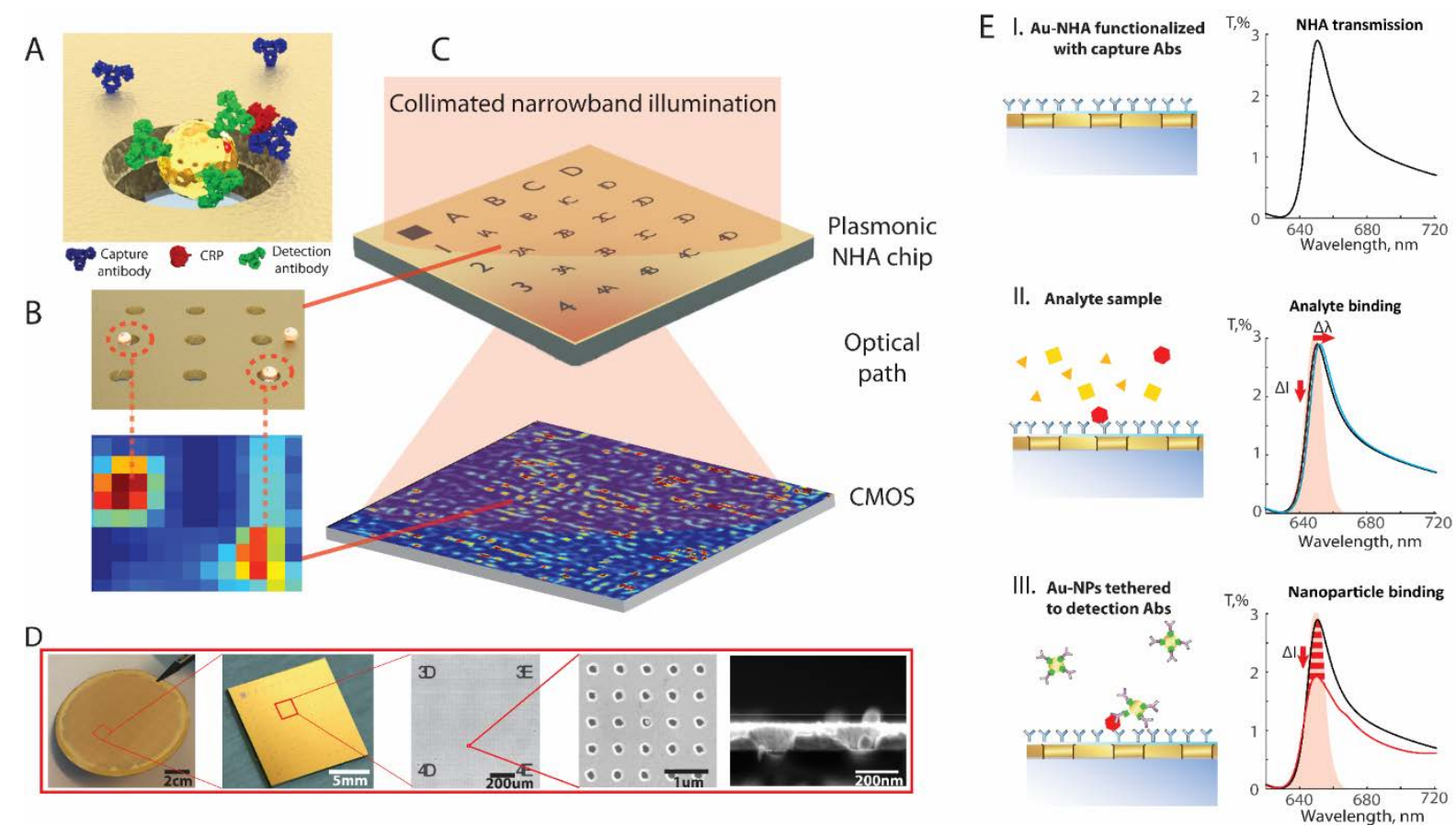

Figure 1. Nanoparticle enhanced plasmonic imager for digital biomarker detection. (A) Antigen (red) is recognized by capture antibodies (blue) immobilized on the Au-NHA and then by detection antibodies (green) tethered to Au-NPs. (B) Strong local suppression in the transmission by Au-NPs creates intensity dips (i.e. red spots) at the corresponding locations of the captured image. (C) Bright-field imaging set-up under narrow-band illumination at the Au-NHA EOT resonance to detect distortions in transmission from plasmonic NHA chip. For accurate image processing, the biosensor chip is patterned with microarray labels. The CMOS camera displays transmission signal as an image heatmap enabling digital detection of biomolecules. (D) Left to Right: Au-NHA wafer with $\mathbf{5 0}$ sensor chips robustly fabricated using scalable low-cost techniques. Au-NHAs uniformly cover the entire plasmonic sensor surface. SEM of a post-patterned chip with microarray marks. SEM of Au-NHAs ( $D=200 \mathrm{~nm}, \mathrm{P}=\mathbf{6 0 0} \mathrm{nm}$ ) with a single Au-NP bound in a hole. Side-view SEM of two neighboring nanoholes with a NP inside one of the nanoholes. (E) I. Simulated transmission spectrum of Au-NHAs exhibiting EOT peak at $650 \mathrm{~nm}$ in air. II. Biomolecule binding causes minuscule spectral shifts in peak position. III. Binding of Au-NPs to the Au-NHA surface results in strong local suppression of the EOT peak. 

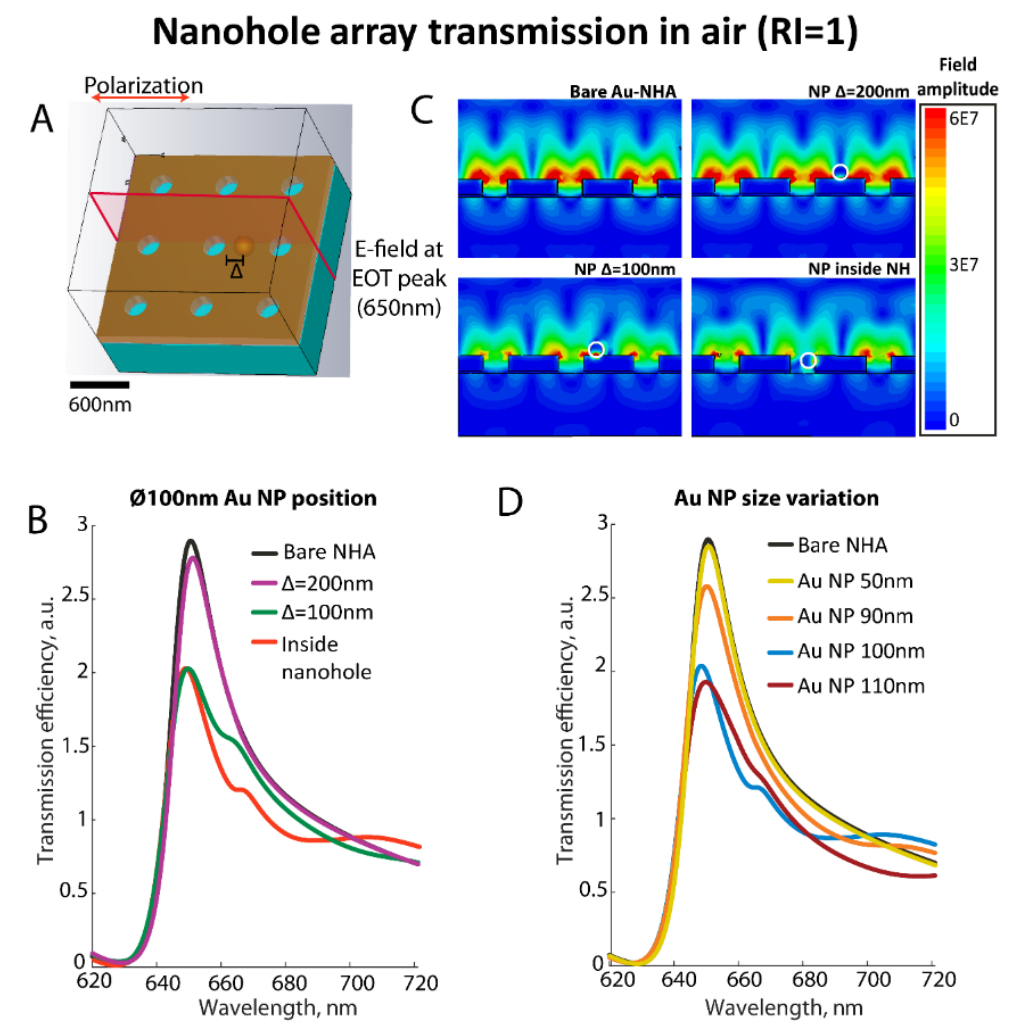

Figure 2. Simulated transmission spectra and electric near-field maps of the Au-NHAs distorted by Au-NPs. (A) Unit cell of $3 \times 3$ nanohole array $(D=200 \mathrm{~nm}, P=600 \mathrm{~nm})$ used in the FEFD simulations excited from top with TM mode and using periodic boundary conditions. (B) Au-NP position dependent EOT transmission spectra of Au-NHAs. The peak suppression increases when Au-NP is bound inside the NH or close to the NH rim and is minimally affected when placed far from the NH. (C) Cross-sections of Au-NHA E-field maps at the EOT resonance $(650 \mathrm{~nm})$. When Au-NPs are inside or close to the NH the LSP modes are disturbed strongly, locally suppressing the transmission. (D) The transmission suppression increases with the NP diameter, saturating at 100 $\mathrm{nm}$. 

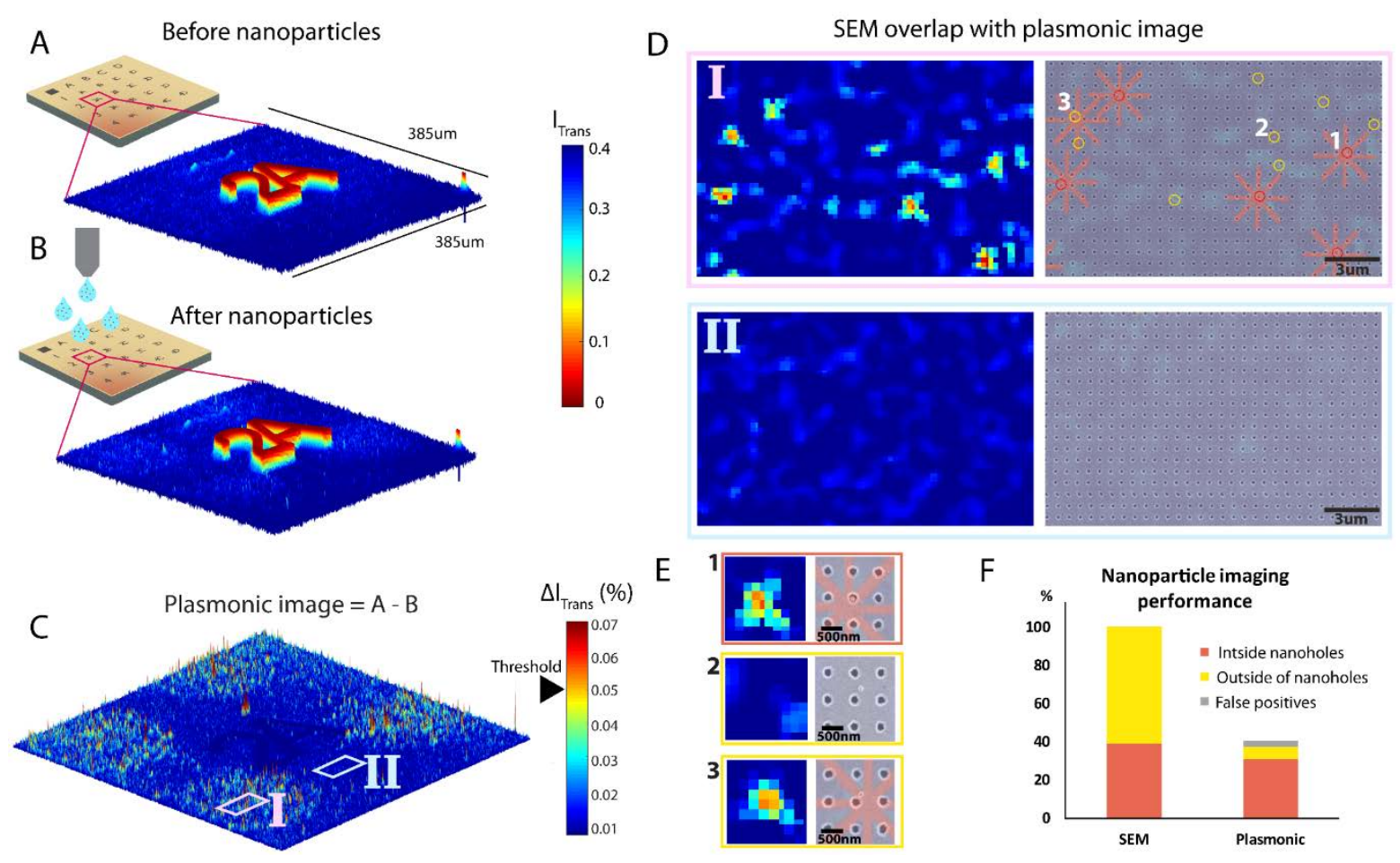

Figure 3. Bright-field plasmonic imaging of Au NPs and SEM validation. (A) Reference images of bare Au-NHAs with alignment microarray labels. (B) Au-NHAs imaged after Au-NPs binding at the same microarray elements shown in (A). (C) The microarray field contains $2 \times 2$ arrays of sensing spots. Color-coded surface plot of transmission intensity spikes caused by NPs formed by computational alignment and subtraction of (B) from (A) produce an image heatmap. (D) Left: Extracted plasmonic images of Au-NHA areas. Right: Plasmonic images overlapped with the SEMs of identical regions with NPs (I) and without NPs (II), zoomed out from (C). The NPs inside NHs are marked by red circles and NPs outside NHs with yellow circles. (E) Au-NPs inside nanoholes produce strong intensity changes (1) and can be detected. NPs far from NHs cannot be detected by plasmonic imaging (2). A fraction of NPs bound close to the NHs rim produces a sufficient intensity change to be identified by the plasmonic imaging (3). (F) In the experiments statistically $\mathbf{4 0} \%$ of NPs are bound inside and $60 \%$ outside of NHs (1000 NPs sample size, verified by SEM). Plasmonic imaging correctly identifies around $40 \%$ of NPs, while false positive signals are $3 \%$ (sample of 127 NPs). 


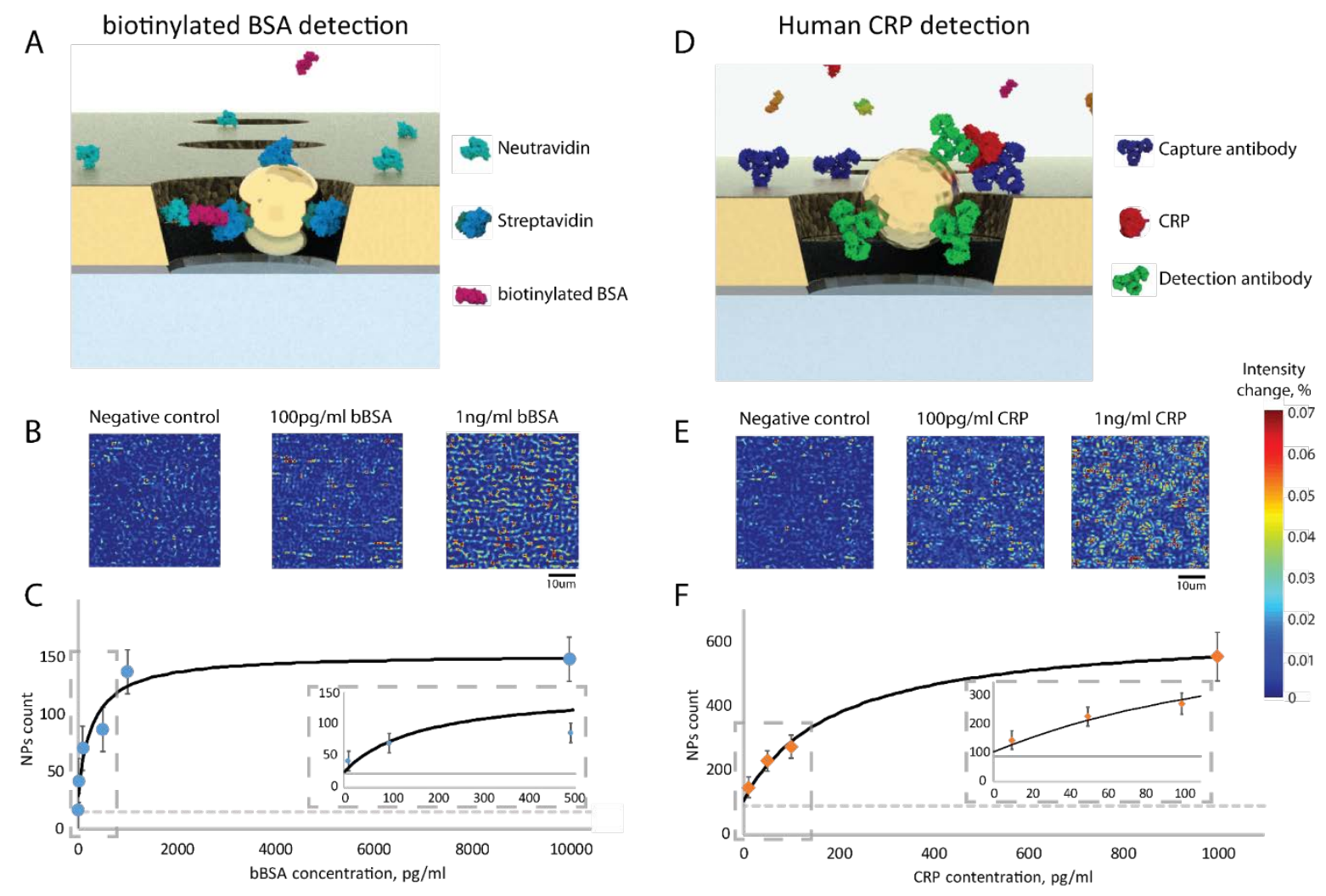

Figure 4. Nanoparticle enhanced digital protein detection. (A) Illustration of bBSA sandwich assay. (B) Different concentrations of bBSA are detected as different densities of NPs are seen on plasmonic heatmap images. (C) bSBA calibration curve (LOD = $10 \mathrm{pg} / \mathrm{ml}$ ). (D) Human CRP sandwich assay. (E) Different concentrations of CRP can be visually distinguished on plasmonic imaging. (F) Human CRP calibration, (LOD $=27 \mathrm{pg} / \mathrm{ml})$. 